\title{
Escleromalacia intercalar espontánea bilateral
}

\section{Sponaneous intercalary scleromalacia}

\author{
Carmen Alba-Linero*, M. Teresa Sainz-de la Maza, Víctor Llorenç-Belles y Alfredo Adán-Civera \\ Departamento de Oftalmología, Hospital Clínic, Barcelona, España
}

\section{Resumen}

Objetivo: Describir un caso clínico de escleromalacia intercalar espontánea a través de fotografías del polo anterior y tomografía de coherencia óptica (OCT) del segmento anterior. Métodos: Una paciente de 32 años consultó por epífora bilateral progresiva. Se le realizaron fotografías, OCT del segmento anterior y topografías de ambos ojos, así como examen sistémico y analítica sanguínea con perfil de autoinmunidad. Resultados: Las fotografías mostraban la ausencia de vascularización límbica con adelgazamiento escleral y coloración azulada por impronta del tejido coroideo. La OCT del segmento anterior revelaba el adelgazamiento escleral con microperforación del mismo. Las topografías y la analítica sanguínea resultaron sin hallazgos significativos. Conclusión: La escleromalacia intercalar espontánea es un raro trastorno que consiste en el adelgazamiento escleral progresivo con microperforación del tejido. Diversos grados de esta enfermedad pueden variar entre cuadros asintomáticos o extrusión del contenido ocular.

Palabras clave: Escleromalacia. Microperforación escleral. Trasplante de membrana amniótica.

\begin{abstract}
Objective: To describe a case of spontaneous intercalary scleromalacia with anterior segment photographs and optical coherence tomography. Methods: A 32-year-old woman attended our ophthalmology department due to progressive bilateral epiphora. Anterior segment photographs, optical coherence tomography and topography were performed, as well as a complete blood count with autoimmunity markers. Results: Anterior segment photographs showed absence of limbal vasculature with scleral thinning and bluish discoloration due to visualization of underlying choroidal tissue. Anterior segment OCT confirmed scleral thinning and microperforations. Topographies and blood tests were normal. Conclusion: Spontaneous intercalary scleromalacia is a rare disorder that consists in progressive scleral thinning with microperforation. The presentation of this disorder varies from asymptomatic to extrusion of ocular contents.
\end{abstract}

Key words: Scleromalacia. Scleral microperforation. Amniotic membrane transplantation.

Correspondencia:

${ }^{*}$ Carmen Alba-Linero

Sabino Arana, $1 \quad$ Fecha de recepción: 21-06-2018

08028 Barcelona, España

Fecha de recepción: 21-06-2018

Disponible en internet: 03-07-2019

E-mail: Carmen.alba.linero@gmail.com

DOI: 10.24875/RMO.M19000074

Rev Mex Oftalmol. 2020;94(3):140-142

www.rmo.com.mx 0187-4519/@ 2018 Sociedad Mexicana de Oftalmología. Publicado por Permanyer. Este es un artículo open access bajo la licencia CC BY-NC-ND (http://creativecommons.org/licenses/by-nc-nd/4.0/). 


\section{Introducción}

La escleromalacia intercalar espontánea es un raro trastorno escleral caracterizado por el adelgazamiento escleral progresivo con perforación espontánea no asociada a otras enfermedades sistémicas u oftalmológicas o traumatismos previos.

Esta lesión puede pasar a menudo desapercibida por ser asintomática o tener microperforaciones imperceptibles. A veces, puede diagnosticarse tras epífora de larga evolución o por presentar maculopatía por hipotonía'.

Hay que realizar siempre un estudio sistémico para descartar enfermedades infecciosas o autoinmunes.

El manejo de la escleromalacia intercalar va desde la observación en casos más leves hasta el recubrimiento con parche escleral o membrana amniótica en casos más graves².

Es necesario hacer un seguimiento estrecho mediante fotografías, tomografía de coherencia óptica (OCT) del polo anterior y resonancia magnética'.

\section{Caso clínico}

Una paciente de 32 años de edad acude a la consulta por notar una ampolla en la conjuntiva de ambos ojos acompañada de sensación de epífora. No tenía antecedentes de interés a nivel sistémico ni oftalmológico.

Refiere tener estas lesiones desde hace más de 1 año, aunque nota agravamiento del cuadro tras un parto hace 5 meses.

Presentaba una agudeza visual de 0.8 en su ojo derecho (OD) y de 0.7 en su ojo izquierdo (OI) bajo la siguiente corrección óptica: OD: $-6.25=-1.00 \times 70^{\circ}$ y OI: $-5.50=-2.00 \times 130^{\circ}$.

A la exploración biomicroscópica destacaban la ausencia de vascularización limbar de $360^{\circ}$ y el adelgazamiento escleral de dicha zona perilimbar, con acúmulo de fluido en el espacio subconjuntival perilimbar de ambos ojos. Se observaba también una cierta coloración azulada perteneciente a tejido coroideo por transiluminación, así como reacción papilar leve en la conjuntiva tarsal (Figs. 1 y 2).

La topografía mostraba parámetros de regularidad con un astigmatismo en contra de la regla y paquimetrías de 531 micras y 542 micras, respectivamente.

En la OCT del segmento anterior se apreciaba la ampolla conjuntival con líquido subyacente por microperforación escleral con adelgazamiento del tejido. Se le realizó una analítica completa en la que solo destacaron los niveles límite de eosinofilia (600/microlitro), el perfil de

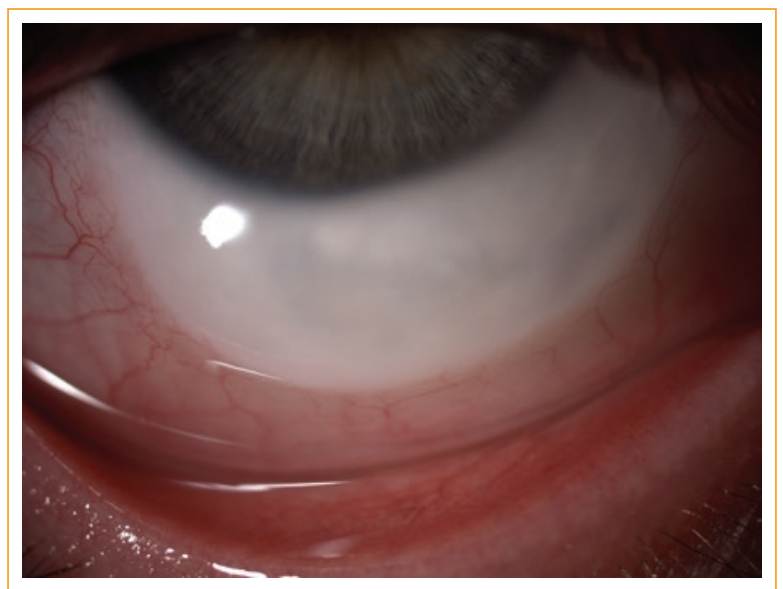

Figura 1. Fotografía del polo anterior del ojo derecho que muestra el adelgazamiento escleral y la ausencia de vasculatura limbar.

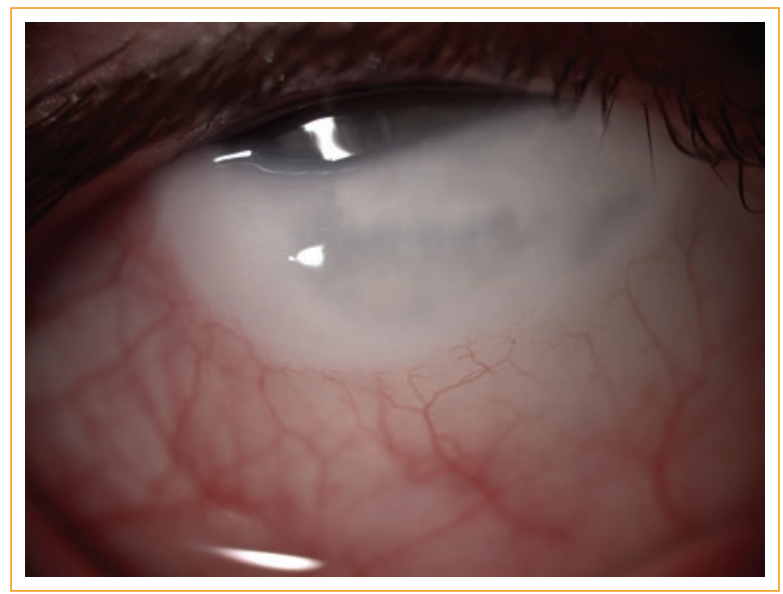

Figura 2. Fotografía del polo anterior del ojo izquierdo que muestra el adelgazamiento escleral y la ausencia de vasculatura limbar, así como reflejo azulado por impronta coroidea.

autoinmunidad (anticuerpos antinucleares, anticuerpos anticitoplasma de neutrófilo, factores de complemento, velocidad de sedimentación glomerular), hemograma y bioquímica resultaron normales (Figs. 3 y 4).

Dada la estabilidad de cuadro en varias visitas consecutivas, se descartó la alternativa quirúrgica mediante parche escleral versus parche de membrana amniótica, y se decidió observación con control mediante OCT.

\section{Discusión}

La esclera mantiene y protege las estructuras oculares $y$, pese a no tener un papel en el procesamiento 


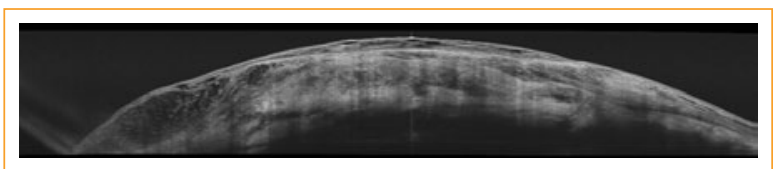

Figura 3. Tomografía de coherencia óptica del polo anterior del ojo derecho que revela el adelgazamiento escleral y la microperforación del tejido.

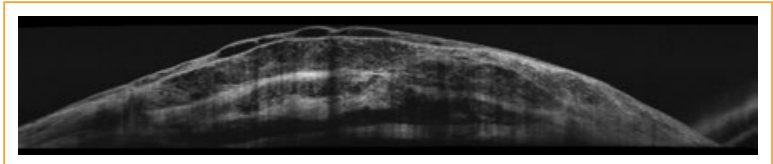

Figura 4. Tomografía de coherencia óptica del polo anterior del ojo derecho que revela el adelgazamiento escleral y la microperforación del tejido.

visual, las enfermedades que le atañen como las escleritis, perforaciones esclerales o miopía patológica suponen un reto terapéutico en oftalmología.

El tejido escleral procede del mesodermo y tiene potencial condrogénico, esto explica que constituya una diana para enfermedades autoinmunes, compartiendo esta característica con el tejido articular; así mismo, la conformación del plexo escleral profundo también contribuye a que el tejido escleral esté afectado en los trastornos de la inmunidad.

La escleromalacia intercalar puede conllevar frecuentemente la perforación espontánea del globo ocular con el consecuente prolapso coroideo ${ }^{4,5}$. Esto supone una urgencia oftalmológica quirúrgica que debe solucionarse en las primeras 24 horas. También se debe explicar a los pacientes que deben evitar deportes de contacto y situaciones que supongan riesgo de traumatismo ocular, dada la susceptibilidad del tejido escleral.

Algunas teorías sostienen que esta enfermedad puede pertenecer al grupo de colobomas oculares ${ }^{6}$ y que se debe a una alteración del colágeno escleral detectado en muestras histopatológicas?.

\section{Conclusión}

Dada que esta rara entidad no tiene una etiopatogenia inflamatoria, no es útil para su manejo el uso de inmunosupresores clásicos o biológicos, como puede suceder en otras enfermedades esclerales, por lo que el manejo de las complicaciones de la perforación escleral intercalar espontánea es quirúrgico.

\section{Conflicto de intereses}

Todos los autores declaran que no existen conflictos de interés.

\section{Responsabilidades éticas}

Protección de personas y animales. Los autores declaran que los procedimientos seguidos se conformaron a las normas éticas del comité de experimentación humana responsable y de acuerdo con la Asociación Médica Mundial y la Declaración de Helsinki.

Confidencialidad de los datos. Los autores declaran que han seguido los protocolos de su centro de trabajo sobre la publicación de datos de pacientes.

Derecho a la privacidad y consentimiento informado. Los autores han obtenido el consentimiento informado de los pacientes y/o sujetos referidos en el artículo. Este documento obra en poder del autor de correspondencia.

\section{Bibliografía}

1. Gaucher D, Ballonzoli L, Saleh M, Sauer A, Bourcier T, Speeg-Schatz C Spontaneous scleral rupture revealed by hypotony maculopathy. 2009; 32,6:438.

2. Ma DH, Wang SF, Su WY, Tsai RJ. Amniotic membrane graft for the management of scleral melting and corneal perforation in recalcitrant infectious scleral and corneoscleral ulcers. Cornea. 2002;21(3):275-83.

3. Seko Y, Azuma N, Takahashi Y, Makino H, Morito T, Muneta T, et al. Human Sclera Maintains Common Characteristics with Cartilage throughout Evolution. PLoS ONE. 2009,3:125-32.

4. Cappin JM, Allen DW. Paralimbic scleromalacia Spontaneous scleral intercalary perforation. Brit. Ophthal. 1973;57:871.

5. Turaga K, Senthil S, Jalali S. Recurrent spontaneous scleral rupture in Marfan's syndrome. BMJ Case Rep. 2016; 2016. pii: bcr2016214764.

6. Fernández López E, Poon A, Huhtanen A, Lindsay R, Green C. Bilateral Blebs Secondary to Spontaneous Scleral Perforations. J Glaucoma. 2017;26,6:177-9.

7. Sorensen TB. Paralimbal scleromalacia. Socalled spontaneous scleral intercalary perforation. Acta Ophthalmol (Copenh). 1975;53,6:901-7. 